\title{
Anterior Cingulate Desynchronization and Functional Connectivity with the Amygdala During a Working Memory Task Predict Rapid Antidepressant Response to Ketamine
}

\author{
Giacomo Salvadore*,', Brian R Cornwell', Fabio Sambataro², David Latov', Veronica Colon-Rosario', \\ Frederick Carver ${ }^{3}$, Tom Holroyd ${ }^{3}$, Nancy DiazGranados', Rodrigo Machado-Vieira', Christian Grillon', \\ Wayne C Drevets ${ }^{4}$ and Carlos A Zarate, Jr'
}

'Experimental Therapeutics, Mood and Anxiety Disorders Program, National Institute of Mental Health, National Institutes of Health, Bethesda, MD, USA; ${ }^{2}$ Gene, Cognition, and Psychosis Program, National Institute of Mental Health, National Institutes of Health, Bethesda, MD, USA; ${ }^{3}$ Magnetoencephalography Core Facility, National Institute of Mental Health, National Institutes of Health, Bethesda, MD, USA; ${ }^{4}$ Section of Neuroimaging in Mood and Anxiety Disorders, Mood and Anxiety Disorders Program, National Institute of Mental Health, National Institutes of Health, Bethesda, MD, USA

\begin{abstract}
Pregenual anterior cingulate cortex (pgACC) hyperactivity differentiates treatment responders from non-responders to various pharmacological antidepressant interventions, including ketamine, an N-methyl-D-aspartate receptor antagonist. Evidence of pgACC hyperactivition during non-emotional working memory tasks in patients with major depressive disorder (MDD) highlights the importance of this region for processing both emotionally salient and cognitive stimuli. However, it is unclear whether pgACC activity might serve as a potential biomarker of antidepressant response during working memory tasks as well, in line with previous research with emotionally arousing tasks. This study tested the hypothesis that during the N-back task, a widely used working memory paradigm, low pretreatment pgACC activity, as well as coherence between the PgACC and the amygdala, would be correlated with the clinical improvement after ketamine. Magnetoencephalography (MEG) recordings were obtained from 15 drug-free patients with MDD during working memory performance I to 3 days before receiving a single ketamine infusion. Functional activation patterns were analyzed using advanced MEG source analysis. Source coherence analyses were conducted to quantify the degree of long-range functional connectivity between the pgACC and the amygdala. Patients who showed the least engagement of the pgACC in response to increased working memory load showed the greatest symptomatic improvement within $4 \mathrm{~h}$ of ketamine administration $(r=0.82, p=0.0002$, false discovery rate (FDR) $<0.05)$. Pretreatment functional connectivity between the pgACC and the left amygdala was negatively correlated with antidepressant symptom change $(r=-0.73, p=0.0021$, FDR $<0.05)$. These data implicate the pgACC and its putative interaction with the amygdala in predicting antidepressant response to ketamine in a working memory task context.

Neuropsychopharmacology (2010) 35, I4I5-1422; doi: I0.1038/npp.2010.24; published online 10 March 2010
\end{abstract}

Keywords: major depressive disorder (MDD); magnetoencephalography (MEG); N-back; biomarker; beta desynchronization

\section{INTRODUCTION}

Major depressive disorder (MDD) is the third leading cause of the global disease burden and a leading cause of disability worldwide (World Health Organization, 2008). Currently available treatments are insufficient for many patients and, even for those patients who do respond favorably, there is a significant therapeutic lag before clinical benefits appear (Trivedi et al, 2006). The finding that a single intravenous

\footnotetext{
*Correspondence: Dr G Salvadore, Experimental Therapeutics, Mood and Anxiety Disorders Program, National Institute of Mental Health, National Institutes of Health, Bethesda, MD, 20892, USA, Tel: + 3014029357, Fax: + 301594 9959, E-mail: salvadoreg@mail.nih.gov Received 30 October 2009; revised 29 January 2010; accepted I February 2010
}

infusion of the $\mathrm{N}$-methyl-D-aspartate antagonist ketamine induces a relatively sustained (ie, lasting 7-10 days) robust antidepressant response within hours has led to exciting new research into the cellular mechanisms and neurocircuitry involved in this rapid onset of antidepressant action (Deakin et al, 2008; Mathew et al, 2008; Price et al, 2009; Zarate et al, 2006). Like with conventional antidepressants, however, ketamine administration does not improve symptoms in all MDD patients, and it is therefore critical to identify neurobiological predictors of treatment response.

We recently found using magnetoencephalography (MEG) that increased pretreatment cortical activity to presentation of affectively charged stimuli (eg, fearful faces) in the anterior cingulate cortex (ACC) predicted antidepressant response to ketamine $4 \mathrm{~h}$ later (Salvadore et al, 
2009). The peak correlation observed was situated anterior to the genu of the corpus callosum, a subregion referred to as the pregenual ACC (pgACC) and theorized to modulate endocrine, autonomic, and experiential aspects of affective states (Drevets et al., 2008) based in part on its functional interaction with limbic structures such as the amygdala (DeRubeis et al., 2008). Given previous data from resting state positron emission tomography and functional magnetic resonance imaging (fMRI) showing that pretreatment pgACC activity predicts the efficacy of a variety of somatic antidepressant interventions, from selective serotonin reuptake inhibitors to repetitive transcranial magnetic stimulation (Mayberg, 2009), the functional integrity of this region may be critical to symptom improvement after intervention, especially given that lower ACC activity either at rest or during emotionally arousing tasks is associated with poor treatment outcome (Mayberg et al, 1997).

Dysfunction of the pgACC in MDD may manifest in others ways, such as showing hyperactivity during tasks that should not typically engage emotional systems or require modulation of affective processes. For instance, Rose et al showed cognitive load-dependent pgACC hyperactivity in patients with MDD using the N-back working memory task (Rose et al, 2006). This hyperactivity was recently shown to be a possible trait marker of depression insofar as it persists after clinical recovery (Schoning et al, 2009). Although the N-back task may be characterized as a mild cognitive stressor (Critchley et al, 2000), it does not involve exposure to emotionally arousing stimuli and thus hyperactivity of the pgACC may be considered aberrant. Accordingly, we aimed to show that pgACC activity in a non-emotional context such as the $\mathrm{N}$-back task has prognostic value as a putative biomarker of antidepressant response just as it does in an emotionally arousing context (Salvadore et al., 2009).

We administered the N-back task to 15 drug-free patients with MDD while MEG recordings were taken, 1 to 3 days before they received a single intravenous infusion of ketamine. Functional patterns of activation were analyzed using advanced MEG source analyses (ie, synthetic aperture magnetometry (SAM) (Hillebrand et al, 2005). The MEG source analysis was restricted to beta frequency activity (ie, $14-30 \mathrm{~Hz}$ ) because previous research with the same N-back task found that power in this frequency band decreasessuggesting desynchronized neural population activity-as memory load increases in the very same fronto-parietal cortical network in which the blood-oxygen-level-dependent responses increase during fMRI studies (Bassett et al, 2009; Coppola et al, 2004; Singh et al, 2002). We hypothesized that with increasing memory load on the N-back task, less engagement of the pgACC would be associated with greater antidepressant response to ketamine. Source coherence imaging analyses (dynamic imaging of coherent sources (DICS) (Gross et al, 2001)) were subsequently performed to quantify long-range coherence or functional connectivity between pgACC and bilateral amygdalae.

\section{PATIENTS AND METHODS}

\section{Participants}

Fifteen patients with a diagnosis of MDD, currently depressed without psychotic features were enrolled in this study; diagnosis was confirmed by the Structured Clinical Interview for Axis I DSM-IV Disorders-Patient Version (First et al., 2002). All subjects were studied at the National Institute of Mental Health Clinical Research Center Mood Disorders Research Unit in Bethesda, MD, USA, between January 2007 and September 2008. Seven out of 15 subjects participated in our previously published study involving the presentation of affectively charged stimuli (Salvadore et al., 2009). Inclusion criteria were a Montgomery-Asberg Depression Rating Scale (MADRS) (Montgomery and Asberg, 1979) score of at least 22, a current or past history of lack of response to two adequate antidepressant trials (operationally defined using the Antidepressant Treatment History Form, Sackeim, 2001), and a current major depressive episode of at least 4 weeks duration. Patients with a DSM-IV (American Psychiatric Association, 1994) diagnosis of drug or alcohol dependence or abuse within the past 3 months, serious, unstable illness, or uncorrected hypo- or hyperthyroidism were excluded. All subjects had been drug free from any psychotropic drugs for at least 2 weeks (or 5 weeks for fluoxetine). The patients had a mean age of $50.5 \pm 13.1$ years, and mean baseline MADRS scores of $33 \pm 4.9$. Sixty percent $(9 / 15)$ had a current comorbid anxiety disorder. MEG recordings were obtained at the end of the drug-free period, 1 to 3 days before the ketamine infusion. The study was approved by the Combined Neuroscience Institutional Review Board at the National Institutes of Health. All subjects provided written informed consent.

\section{Ketamine Administration}

Ketamine infusion was similar to a previously described procedure (Zarate et al, 2006). Briefly, MDD patients openly received a single intravenous infusion of saline solution and $0.5 \mathrm{mg} / \mathrm{kg}$ of ketamine hydrochloride (Abbott Laboratories, Abbott Park, IL) over the course of $40 \mathrm{~min}$. Symptoms were reassessed 230 min after ketamine infusion, as described in our previous research (Phelps et al, 2009; Salvadore et al, 2009). This represented an ideal time point for analyzing early antidepressant response given that in our previous placebo-controlled study most of the patients who responded to ketamine $(88 \%)$ did so by $230 \mathrm{~min}$ (Zarate et al, 2006). Baseline and post-ketamine scores for depressive, anxiety, and psychotic symptoms were obtained using the MADRS, the Hamilton Anxiety Scale (HAM-A) (Hamilton et al, 1959), and the Brief Psychiatric Rating Scale (BPRS)positive symptoms subscale (Overall and Gorham, 1962). Changes in psychiatric symptoms were expressed as percentage change from baseline according to each individual's scores, with positive percentages reflecting a reduction in symptoms. Post-assessment of anxiety symptoms was missing for two patients.

\section{N-Back Task}

The N-back task is a working memory task that demands simultaneous encoding, maintenance, and retrieval of stimuli and has been described in greater detail elsewhere (Callicott et al, 1999). Participants responded to presentations of one of four numbers (0.5-s duration, 1.8-s intertrial 
interval) by pressing a button to match the current stimulus (0-back) or the number presented either one or two trials previously. Subjects performed a run of 18 blocks, with each block lasting $22 \mathrm{~s}$, alternating between a 2-back, a 1-back, and a 0 -back task. Subjects performed an N-back practice run before the actual recording, as described by Callicott et al (Callicott et al, 1999).

\section{Data Acquisition}

MEG recordings were digitized at $1200 \mathrm{~Hz}$ with a $0-300 \mathrm{~Hz}$ bandwidth using a CTF 275 MEG system (CTF Systems, Coquitlam, Canada). The whole-head MEG system comprises an array of 275 radial first-order gradiometers housed in a magnetically shielded room (Vacuumschmelze, Hanau, Germany). Background noise was removed online through active noise cancellation using synthetic third-gradient balancing. In a separate session using a $1.5 \mathrm{~T}$ or a $3 \mathrm{~T}$ GE scanner (Milwaukee, WI), anatomical MRIs were obtained for coregistration with the MEG data.

\section{Source Power Analysis}

SAM (Hillebrand et al, 2005; Vrba and Robinson, 2001) was used to estimate neural oscillatory power differences in the beta band $(14-30 \mathrm{~Hz})$ across two different load conditions, 2-back vs 1-back and 2-back vs 0-back. SAM uses signal covariance across the entire sensor array to construct an optimum spatial filter at any source point. It is optimized to suppress all activity except at the location of interest in source space by minimizing the output of the filter with the constraint of unity pass gain at the location of interest (based on a multi-sphere head model derived from each MRI). Volumetric images of source power can be produced by constructing spatial filters at each point in a three-dimensional grid encompassing the brain. Covariance matrices were calculated over $500 \mathrm{~ms}$ epochs centered on the subject's response. Dual-state images were produced using the pseudo-F ratio statistic with a $7.5 \mathrm{~mm}$ step size, directly contrasting two conditions (eg, 2-back vs 1-back). This was repeated for each of the two comparisons.

SAM source images were manually coregistered to participants' MRIs using AFNI software (Cox, 1996). A within-volume normalization procedure was applied to each source image to compensate for inter-subject variability in global power. For group analyses, all source images were spatially warped into the stereotaxic array of Talairach and Tournoux (Talairach and Tournoux, 1988). Pearson correlation analyses were conducted to determine whether beta oscillatory power ratios for any of the load contrasts predicted change in depressive symptoms after ketamine administration. Given our a priori hypothesis that ACC activity related to cognitive load would predict antidepressant response to ketamine, we estimated false discovery rates (FDRs), (Genovese et al, 2002) within a region of interest (ROI) approach encompassing the ACC/BA24/32 (ie, voxel-wise comparisons were performed within an ROI of volume $=1847.5 \mathrm{~mm}$ isotropic voxels). Correlation statistics within this ROI were considered significant at an FDR threshold of $<0.05$.

\section{Source Coherence Analysis}

To analyze brain regions whose interaction with the ACC might be relevant for predicting treatment response to ketamine, we used DICS (Gross et al, 2001). DICS is a spatial-filtering approach in the frequency domain that can be extended to localize coherent brain neuronal populations (ie, functional connectivity). With this method, projected source coherence between a selected seed point and all other points across source space is estimated; as a measure of association in the frequency domain, coherence does not indicate directionality nor whether two regions are directly interacting rather than, for instance, both being driven by a third region. Self-coherence between neighboring points because of non-orthogonality of the spatial filters is suppressed by computing contrasts of different conditions with the same seed point (eg, 2-back vs 1-back) to produce differential coherence values.

All parameters (eg, time window, frequency band, voxel size) and preprocessing steps were identical to those used for the SAM analyses. A single seed point in the pgACC was selected for coherence analysis; it was based on the SAM source results indicating the locus of maximum correlation between beta oscillatory power and antidepressant response. Pearson correlation analyses were performed to establish whether the degree of differential coherence between the pgACC and amygdala also predicted antidepressant response after administration of ketamine. An ROI encompassing bilateral amygdalae was applied and correlation statistics within this ROI were considered significant at an FDR threshold of $<0.05$.

\section{RESULTS}

\section{Treatment Response}

Depressive symptoms were significantly improved $230 \mathrm{~min}$ after the infusion, as assessed by change in MADRS score $(t(14)=4.81, p<0.001$ (mean MADRS pretreatment score $33.5 \pm 4.9$; mean MADRS score $230 \mathrm{~min}$ after ketamine $21.0 \pm 11.6))$. A significant decrease was also observed in both anxiety symptoms (mean HAM-A pretreatment score $23.8 \pm 5.7$; mean HAM-A score after $230 \mathrm{~min} 13.6 \pm 5.4$; $t(12)=5.94, p<0.001$ ), and psychotic symptoms (mean BPRS-positive subscale pretreatment score 10.2 \pm 1.6 ; mean BPRS-positive symptoms score after $230 \mathrm{~min} 8.9 \pm 1.1$ : $t(14)=3.08, p<0.01)$.

\section{Behavioral Results}

Increasing working memory load was associated with decreased accuracy, as expected on the N-back task (Callicott et al, 1999) (mean accuracy: 0-back: $98.7 \pm 1.5 \%$; 1-back: $93.1 \pm 9.2 \%$; 2-back: $81.6 \pm 10.0 \% ; \quad F=16.955$; $p<0.001 ; \mathrm{df}=2,28)$.

\section{Correlation Between ACC Activity and Symptom Change After Ketamine}

Pretreatment pregenual and subgenual ACC beta desynchronization for the 2-back vs 1-back comparison was positively correlated with antidepressant response $230 \mathrm{~min}$ 


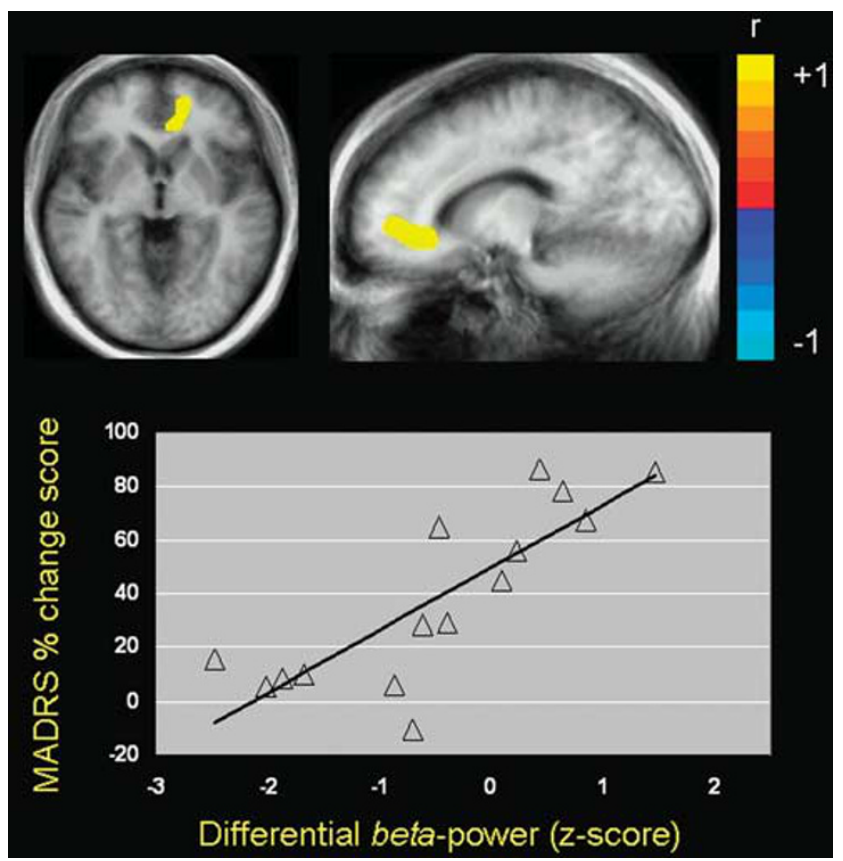

Figure I Pearson correlation between beta desynchronization in the anterior cingulate cortex (ACC) and change in depressive symptoms 230 min after ketamine infusion for the 2-back vs I-back comparison in patients with MDD (ACC peak: $x=-15, y=45, z=-1 \mathrm{~mm}$; coordinates expressed according to the stereotaxic atlas of Talairach and Tournoux (Talairach and Tournoux, 1988)). These coordinates localize to the pregenual portion of the ACC, although the cluster of voxel $t$-values extends into adjacent subgenual ACC. Images are in radiological orientation (left $=$ right, right $=$ left) and are thresholded at FDR $<0.05$

after ketamine infusion, with a local maximum in the left pgACC (peak in Talairach coordinates: $-15,45,-1 \mathrm{~mm}$, $r(13)=0.82, p=0.0002$, FDR $<0.05$ ) (Figure 1). Patients who showed the greatest beta desynchronization in this region were the least likely to respond to ketamine, whereas patients who showed relatively little change in beta activity showed the highest likelihood of antidepressant response. We also observed a similar relationship between pgACC beta desynchronization and reduced anxiety symptom severity, as assessed with the HAM-A rating scale, in the same peak locus as antidepressant response $(r(11)=0.88$, $p<0.0001$, FDR $<0.05)$. No such relationship was observed between pretreatment ACC activity and antidepressant response for the 2-back vs 0-back comparison. However, when more liberal statistical criteria were applied, we found a cluster of several voxels in the right pgACC that predicted antidepressant response to ketamine for the 2-back $v s$ 0-back comparison (peak: $-9,45,6 \mathrm{~mm}, \mathrm{r}(13)=0.66$, $p<0.01$, uncorrected)

\section{Functional Connectivity Analysis and Symptom Change After Ketamine}

Pretreatment functional connectivity (ie, beta coherence) between the pgACC and the left amygdala for the 2-back $v s$ 1-back comparison was negatively correlated with antidepressant symptom change after ketamine infusion (peak: $-30,-7,-16 \mathrm{~mm}, r(13)=-0.73, p=0.0021$, FDR $<0.05)$ (Figure 2). Specifically, subjects who showed the least

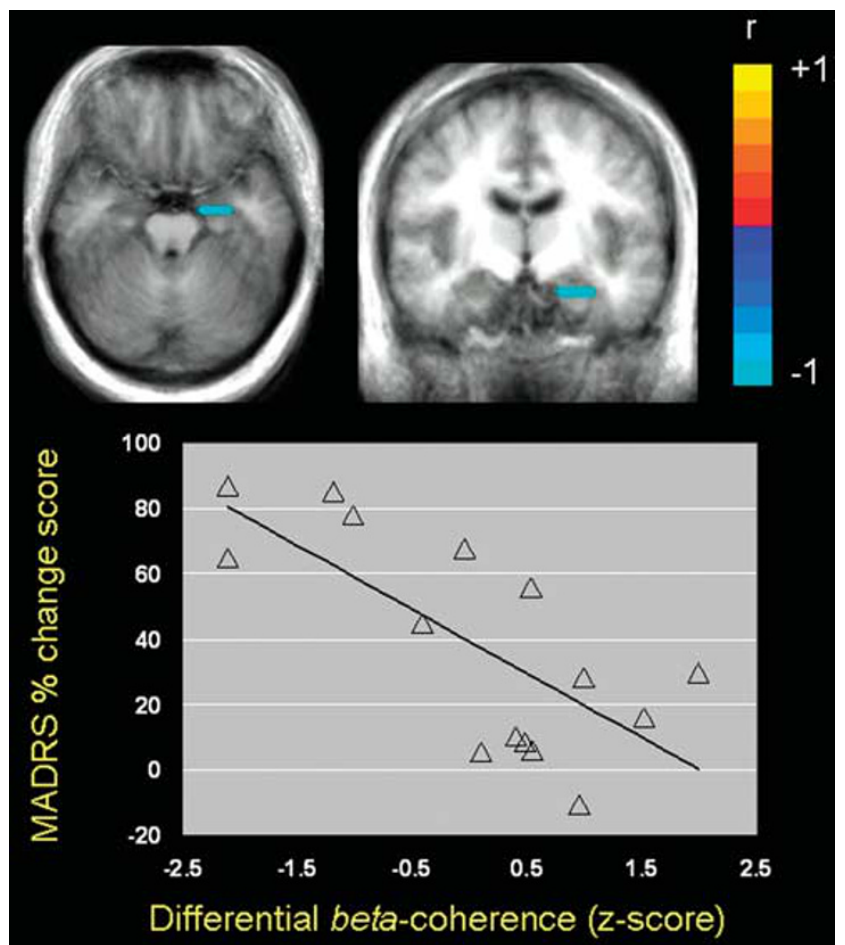

Figure 2 Pearson correlation between differential source coherence of the PgACC with the left amygdala and change in depressive symptoms 230 min after ketamine infusion for the 2-back vs I-back comparison in patients with MDD (left amygdala peak: $x=-30, y=-7, z=-16 \mathrm{~mm}$; coordinates expressed according to the stereotaxic atlas of Talairach and Tournoux (Talairach and Tournoux, 1988)). Images are in radiological orientation (left $=$ right, right $=$ left) and are thresholded at FDR $<0.05$.

coherence between pgACC and left amygdala beta activity were those who showed the greatest antidepressant response to ketamine. Coherence between pgACC and the amygdala did not predict decreased anxiety symptoms after ketamine infusion (peak: $-30,-7,-16 \mathrm{~mm}, r(13)=-0.53$ $p>0.05$, uncorrected).

\section{DISCUSSION}

In this study, we found that pretreatment pgACC activity during N-back task performance was correlated with the clinical improvement after ketamine in patients with MDD. Subjects who showed the least engagement of the pgACC with increased working memory load showed the greatest symptomatic improvement within $4 \mathrm{~h}$ of ketamine administration. This relationship was found for the 2-back vs 1-back contrast but not when comparing the 2-back to the 0 -back.

We previously found that increased activity in the pgACC in response to faces showing negative emotional expressions was positively correlated with rapid antidepressant response to ketamine; the reverse relationship was found with activity in the right amygdala (Salvadore et al, 2009). It is noteworthy that our present data implicate the same brain region as a putative biomarker of treatment response to ketamine during a cognitively demanding task. For ease of comparison, we present the extent of spatial overlap between the present data and those reported previously 


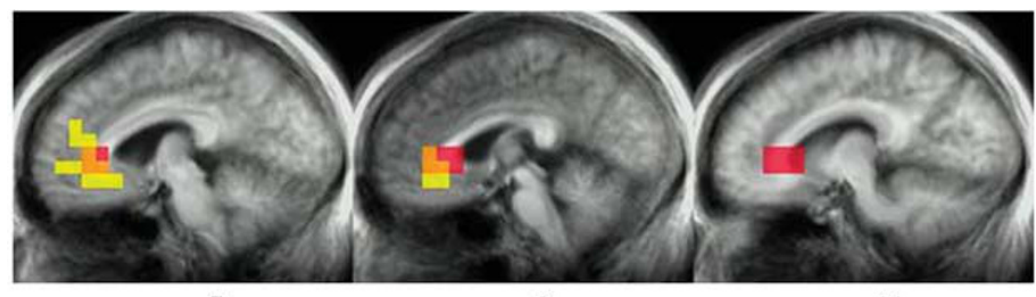

$x=-5$

$x=3$ $x=11$

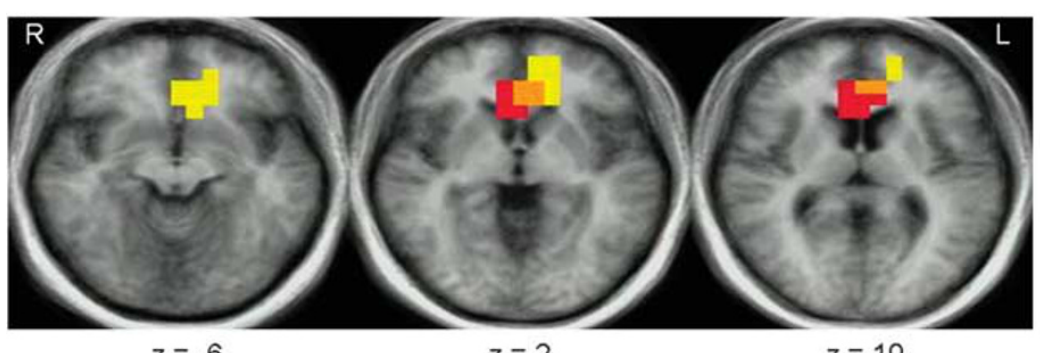

N-back

$z=-6$

$z=2$

$z=10$

Figure 3 Overlapping region of the ACC that shows activity correlated with antidepressant response to ketamine across two studies (in orange). This

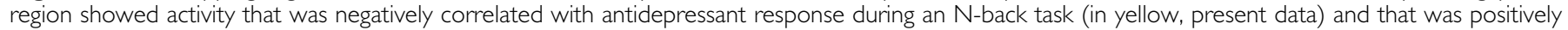
correlated with antidepressant response during exposure to negative emotional faces (in red, from Salvadore et al, 2009).

(Salvadore et al, 2009) (Figure 3). As higher beta desynchronization with increasing memory load reflects greater ACC engagement, our results imply that the pgACC shows opposite changes in activity in emotional vs nonemotional cognitive task contexts in MDD patients who respond favorably to ketamine. Taken together, these findings suggest that high pgACC activity in response to emotionally salient stimuli, but low pgACC activity in response to increased cognitive demands, predicts antidepressant response to ketamine.

These findings concur with results from functional studies in healthy volunteers that show that the pgACC shows both increased and decreased blood flow depending on the emotionally arousing and cognitive demand features of the task (reviewed in Drevets and Raichle, 1998); for example, pgACC regional blood flow increases during emotionally salient tasks, such as induction of sadness (George et al, 1995), but decreases during cognitive tasks that demand attention (deactivation), including visuospatial and working memory tasks (Corbetta et al, 1991; Rose et al, 2006). In light of this evidence, our results appear to suggest that preservation of this normative pattern predicts better treatment outcome.

Pretreatment ACC activity is a putative biomarker of treatment response to several antidepressant interventions with different mechanisms of action, including nortriptyline, SSRIs, cognitive behavioral therapy, and sleep deprivation (reviewed in Mayberg, 2009). It would thus be premature to conclude that the prognostic value of measuring pgACC activity carries specificity with regard to particular treatments such as ketamine. Pretreatment pgACC activity may simply indicate general proneness to show clinical improvement independently of the type of intervention. Nevertheless, it is difficult assimilating our findings to those of previous studies because while we enrolled patients with some degree of treatment resistance, other studies investigating pretreatment predictors of antidepressant response did not require any degree of treatment resistance as an inclusion criterion. Future studies comparing ketamine to conventional antidepressants in the same patient sample will be able to address these issues.

Using a similar working memory task, Walsh et al implicated the dACC, rather than the pgACC, as a predictor of treatment response to fluoxetine in patients with MDD (Walsh et al, 2007); patients who showed higher engagement of the dACC as working memory load increased showed worse treatment response. Although the dACC and the pgACC are thought to be functionally and pathophysiologically independent, the results from this study are not at odds with the findings reported by Walsh et al. Indeed, we found a similar relationship between pretreatment ACC activity and treatment response, that is, an inverse correlation between ACC activity and symptom change. Furthermore, a recent study by Schöning et al (Schoning et $a l, 2009$ ) in patient with remitted depression showed evidence of hyperactivity in both the pgACC and the dACC, implying a similar function of those two areas in the context of working memory processing, at least in patients.

Whether the role of the ACC as a predictor of clinical response to different antidepressant interventions seems now established, less clear is the role of the dorsolateral prefrontal cortex (DLPFC); indeed, cross-sectional working memory studies in MDD patients compared with healthy controls, which used the N-back task yielded conflicting evidence, reporting both no difference (Barch et al, 2003; Rose et al, 2006) and increased (Harvey et al, 2005; (Matsuo et al, 2007) DLPFC activity in depressed patients. On the basis of the existing literature, we might argue that DLPFC activity is more a target of antidepressant drugs in patients who do respond to treatment (Brody et al, 1999; Kennedy et al, 2007) rather than a predictor of efficacy (see DeRubeis et $a l, 2008$ for an excellent review addressing the role of the DLPFC in treatment response in depression).

Moreover, we found that subjects who showed the lowest source coherence between the pgACC and the left amygdala 
were the most likely to respond to ketamine. This was observed for the 2-back vs 1-back, but not for the 2-back $v s$ 0 -back comparison. Decreased functional connectivity between those two regions might reflect decreased subcortical activation and therefore less need for cortical control over the amygdala in those who respond to ketamine. Indeed, subjects with the highest coherence had the lowest treatment response. Thus, these data might indicate that ketamine responders show functional integrity of the cortico-limbic mood regulating circuit, which is not actively engaged during cognitively demanding tasks, but that non-responders show abnormal activation of mood regulating circuitry even in the absence of emotionally arousing stimuli. Complementary evidence has been reported of increased functional connectivity between these two regions in response to negative $v s$ neutral pictures after treatment with sertraline (Anand et al, 2005) and fluoxetine (Chen et al, 2008).

This study had several limitations. The sample size was relatively small, but in line with previous imaging studies, which looked at putative biomarkers of treatment response with conventional antidepressants (Kennedy et al, 2007; Langenecker et al, 2007). As a result of the small sample size, a direct comparison between responders and non-responders using a categorical definition of response would have likely been underpowered; we, therefore, analyzed the correlation between ACC activity and clinical improvement (or decrease in depressive symptoms) to ketamine using a continuous definition of response, which might also be able to better represent the continuous distribution of the decrease in depression severity after an antidepressant drug trial (MacCallum et al, 2002). Future studies with a larger sample size will be able to investigate whether pgACC activity predicts antidepressant response and remission to ketamine using a categorical approach.

As in our previous study, we enrolled only inpatients with treatment-resistant MDD, and high comorbidity with anxiety disorders, thus the generalizability of our findings to patients with milder depression remains uncertain. ACC beta desynchronization-but not functional connectivity between the pgACC and the amygdala - predicted not only decreased severity of depression but also reduced severity of anxiety symptoms assessed through the HAM-A rating scale; this is not surprising considering the shared pathophysiological determinants of MDD and anxiety disorders. Future studies will need to enroll MDD patients with no Axis I comorbidities to clarify the specificity of the present findings for the depressive component of MDD.

We also observed a significant reduction of BPRS-positive symptoms subscale scores at $230 \mathrm{~min}$ compared with the baseline assessment. This is consistent with the findings from our previous randomized placebo-controlled study, in which we observed the same decrease in BPRS-positive symptoms at $230 \mathrm{~min}$ (Zarate et al, 2006). In that study, a significant increase in the BPRS-positive subscale was observed after $40 \mathrm{~min}$ from the infusion, but returned back to baseline when the first antidepressant effects became apparent (ie $\geqslant 80 \mathrm{~min}$ ). In this study, patients showed low baseline levels of symptoms within the positive BPRS subscale-given the exclusion of patients with psychotic features - and we observed a mean decrease of just 1.2 points in the BPRS symptom psychotic subscale scores at 230 min after the infusions, which might be hardly clinically meaningful.

As we assessed antidepressant response only at $230 \mathrm{~min}$, it also remains unknown whether pgACC activity and pgACC connectivity with the amygdala differentiate treatment responders from non-responders to ketamine at later time points. Furthermore, as the ACC has been implicated as a target of placebo as well as antidepressant response to active treatments (Benedetti et al, 2005), our findings could reflect not only the pharmacological actions of ketamine but also nonspecific placebo effects. However, this hypothesis is unlikely, as in our previous study with a placebo-controlled design, which used the same inclusion criteria as this study just 1 of 12 patients had a placebo response at $230 \mathrm{~min}$ from the infusion.

Finally, it is also unknown whether the pgACC is relevant not only as a potential biomarker of treatment response to ketamine but also as a potential direct target for ketamine's mechanism of action. It is noteworthy that studies in which post-treatment changes in metabolic activity were measured show that the pgACC is also a target of antidepressant treatments, showing decreased activity after paroxetine or venlafaxine (Goldapple et al, 2004; Kennedy et al, 2007) and increased activity after cognitive-behavioral therapy (Kennedy et al, 2007) in patients who respond to treatment. Future placebo-controlled studies of ketamine with a larger sample and a longitudinal design are needed to address these questions.

Taken together, our data strongly implicate the pgACC and its functional connectivity with the amygdala in predicting antidepressant response to ketamine in a nonemotional cognitive task context. The data also provide important insights into the function of the pgACC in working memory processes and the pathophysiology of MDD.

\section{ACKNOWLEDGEMENTS}

This research was supported by the Intramural Research Program of the National Institute of Mental Health, National Institutes of Health, and a National Alliance for Research on Schizophrenia and Depression Award (CZ). Ioline Henter provided invaluable editorial assistance.

\section{DISCLOSURE}

The author(s) declare that, except for income received from our primary employer, no financial support or compensation has been received from any individual or corporate entity over the past 3 years for research or professional service and there are no personal financial holdings that could be perceived as constituting a potential conflict of interest. A patent application for the use of ketamine in depression has been submitted listing Dr Zarate among the inventors. Dr Zarate has assigned all rights on the patent to the US government. The authors declare that over the past 6 months WCD has received compensation from Pfizer Pharmaceuticals, the manufacturer of ketamine. 


\section{REFERENCES}

American Psychiatric Association (1994). Diagnostic and Statistical Manual of Mental Disorders IV. American Psychiatric Association: Washington, DC.

Anand A, Li Y, Wang Y, Wu J, Gao S, Bukhari L et al (2005). Antidepressant effect on connectivity of the mood-regulating circuit: an FMRI study. Neuropsychopharmacology 30: 1334-1344.

Barch DM, Sheline YI, Csernansky JG, Snyder AZ (2003). Working memory and prefrontal cortex dysfunction: specificity to schizophrenia compared with major depression. Biol Psychiatry 53: 376-384.

Bassett DS, Bullmore ET, Meyer-Lindenberg A, Apud JA, Weinberger DR, Coppola R (2009). Cognitive fitness of costefficient brain functional networks. Proc Natl Acad Sci USA 106: 11747-11752.

Benedetti F, Mayberg HS, Wager TD, Stohler CS, Zubieta JK (2005). Neurobiological mechanisms of the placebo effect. J Neurosci 25: 10390-10402.

Brody AL, Saxena S, Silverman DH, Alborzian S, Fairbanks LA, Phelps ME et al (1999). Brain metabolic changes in major depressive disorder from pre- to post-treatment with paroxetine. Psychiatry Res 91: 127-139.

Callicott JH, Mattay VS, Bertolino A, Finn K, Coppola R, Frank JA et al (1999). Physiological characteristics of capacity constraints in working memory as revealed by functional MRI. Cereb Cortex 9: $20-26$

Chen $\mathrm{CH}$, Suckling J, Ooi C, Fu CH, Williams SC, Walsh ND et al (2008). Functional coupling of the amygdala in depressed patients treated with antidepressant medication. Neuropsychopharmacology 33: 1909-1918.

Coppola R, Callicott JH, Holroyd T, Verchinski BA, Sust S, Weinberger DR (2004). MEG activation comparison to fMRI BOLD for a working memory task. Proceedings of the 14th International Conference on Biomagnetism, Boston, 2004, p 7.

Corbetta M, Miezin FM, Dobmeyer S, Shulman GL, Petersen SE (1991). Selective and divided attention during visual discriminations of shape, color, and speed: functional anatomy by positron emission tomography. J Neurosci 11: 2383-2402.

Cox RW (1996). AFNI: software for analysis and visualization of functional magnetic resonance neuroimages. Comput in Biomed Res 29: 162-173.

Critchley HD, Corfield DR, Chandler MP, Mathias CJ, Dolan RJ (2000). Cerebral correlates of autonomic cardiovascular arousal: a functional neuroimaging investigation in humans. J Physiol 523(Pt 1): 259-270.

Deakin JF, Lees J, McKie S, Hallak JE, Williams SR, Dursun SM (2008). Glutamate and the neural basis of the subjective effects of ketamine: a pharmaco-magnetic resonance imaging study. Arch Gen Psychiatry 65: 154-164.

DeRubeis RJ, Siegle GJ, Hollon SD (2008). Cognitive therapy versus medication for depression: treatment outcomes and neural mechanisms. Nat Rev Neurosci 9: 788-796.

Drevets WC, Price JL, Furey ML (2008). Brain structural and functional abnormalities in mood disorders: implications for neurocircuitry models of depression. Brain Struct Funct 213: 93-118.

Drevets WC, Raichle ME (1998). Reciprocal suppression of regional cerebral blood flow during emotional versus higher cognitive processes: implications for interactions between emotion and cognition. Cognit Emot 353-385.

First M, Spitzer RL, Gibbon M, Williams JBW (2002). Structured Clinical Interview for the DSM-IV-TR Axis I Disorders- Patient edition. Biometrics Research Department, New York State Psychiatric Institute: New York, NY.
Genovese CR, Lazar NA, Nichols T (2002). Thresholding of statistical maps in functional neuroimaging using the false discovery rate. Neuroimage 15: 870-878.

George MS, Ketter TA, Parekh PI, Horwitz B, Herscovitch P, Post RM (1995). Brain activity during transient sadness and happiness in healthy women. Am J Psychiatry 152: 341-351.

Goldapple K, Segal Z, Garson C, Lau M, Bieling P, Kennedy S et al (2004). Modulation of cortical-limbic pathways in major depression treatment-specific effects of cognitive behavior therapy. Arch Gen Psychiatry 61: 34-41.

Gross J, Kujala J, Hamalainen M, Timmermann L, Schnitzler A, Salmelin R (2001). Dynamic imaging of coherent sources: studying neural interactions in the human brain. Proc Natl Acad Sci USA 98: 694-699.

Hamilton MC, Schutte NS, Malouff JM (1959). Hamilton Anxiety Scale [HAMA]. In: Hamilton MC (ed). Source Book of Adult Assessment (Applied Clinical Psychology). Plenum Press: New York, pp 154-157.

Harvey PO, Fossati P, Pochon JB, Levy R, Lebastard G, Lehéricy S et al (2005). Cognitive control and brain resources in major depression: an fMRI study using the n-back task. Neuroimage 26: 860-869.

Hillebrand A, Singh KD, Holliday IE, Furlong PL, Barnes GR (2005). A new approach to neuroimaging with magnetoencephalography. Hum Brain Mapp 25: 199-211.

Kennedy SH, Konarski JZ, Segal ZV, Lau MA, Bieling PJ, McIntyre RS et al (2007). Differences in brain glucose metabolism between responders to $\mathrm{CBT}$ and venlafaxine in a 16-week randomized controlled trial. Am J Psychiatry 164: 778-788.

Langenecker SA, Kennedy SE, Guidotti LM, Briceno EM, Own LS, Hooven $\mathrm{T}$ et al (2007). Frontal and limbic activation during inhibitory control predicts treatment response in major depressive disorder. Biol Psychiatry 62: 1272-1280.

MacCallum RC, Zhang S, Preacher KJ, Rucker DD (2002). On the practice of dichotomization of quantitative variables. Psychol Methods 7: 19-40.

Mathew SJ, Manji HK, Charney DS (2008). Novel drugs and therapeutic targets for severe mood disorders. Neuropsychopharmacology 33: 2080-2092.

Matsuo K, Glahn DC, Peluso MAM, Hatch JP, Monkul ES, Naji P et al (2007). Prefrontal hyperactivation during working memory task in untreated individuals with major depressive disorder. Mol Psychiatry 12: 158-166.

Mayberg HS (2009). Targeted electrode-based modulation of neural circuits for depression. J Clin Invest 119: 717-725.

Mayberg HS, Brannan SK, Mahurin RK, Jerabek PA, Brickman JS, Tekell JL et al (1997). Cingulate function in depression: a potential predictor of treatment response. Neuroreport 8: 1057-1061.

Montgomery SA, Asberg M (1979). A new depression scale designed to be sensitive to change. Br J Psychiatry 134: 382-389.

Overall JE, Gorham DR (1962). The brief psychiatric rating scale. Psychol Rep 10: 790-812.

Phelps LE, Brutsche N, Moral JR, Luckenbaugh DA, Manji HK, Zarate Jr CA (2009). Family history of alcohol dependence and initial antidepressant response to an N-methyl-D-aspartate antagonist. Biol Psychiatry 65: 181-184.

Price RB, Knock MK, Charney DS, Mathew SJ (2009). Effects of intravenous ketamine on explicit and implicit measures of suicidality in treatment-resistant depression. Biol Psychiatry 66: $522-526$.

Rose EJ, Simonotto E, Ebmeier KP (2006). Limbic over-activity in depression during preserved performance on the n-back task. Neuroimage 29: 203-215.

Sackeim HA (2001). The definition and meaning of treatmentresistant depression. J Clin Psychiatry 62(Suppl) 16: 10-17.

Salvadore G, Cornwell BR, Colon-Rosario V, Coppola R, Grillon C, Zarate Jr CA et al (2009). Increased anterior cingulate cortical 
activity in response to fearful faces: a neurophysiological biomarker that predicts rapid antidepressant response to ketamine. Biol Psychiatry 65: 289-295.

Schoning S, Zwitserlood P, Engelien A, Behnken A, Kugel H, Schiffbauer $\mathrm{H}$ et al (2009). Working-memory fMRI reveals cingulate hyperactivation in euthymic major depression. Hum Brain Mapp 30: 2746-2756.

Singh KD, Barnes GR, Hillebrand A, Forde EM, Williams AL (2002). Task-related changes in cortical synchronization are spatially coincident with the hemodynamic response. Neuroimage 16: 103-114.

Talairach J, Tournoux P (1988). Co-planar Stereotaxic Atlas of the Human Brain. Thieme Medical: New York.

Trivedi MH, Rush AJ, Wisniewski SR, Nierenberg AA, Warden D, Ritz L et al (2006). Evaluation of outcomes with citalopram for depression using measurement-based care in $\mathrm{STAR}^{\star} \mathrm{D}$ : implications for clinical practice. Am J Psychiatry 163: 28-40.

Vrba J, Robinson SE (2001). Signal processing in magnetoencephalography. Methods 25: 249-271.

Walsh ND, Williams SC, Brammer MJ, Bullmore ET, Kim J, Suckling J et al (2007). A longitudinal functional magnetic resonance imaging study of verbal working memory in depression after antidepressant therapy. Biol Psychiatry 62: 1236-1243.

World Health Organization. (2008). The global Burden of Disease: A 2004 Update.

Zarate Jr CA, Singh JB, Carlson PJ, Brutsche NE, Ameli R, Luckenbaugh DA et al (2006). A randomized trial of an Nmethyl-D-aspartate antagonist in treatment-resistant major depression. Arch Gen Psychiatry 63: 856-864. 